little general interest, because I find that very few are informed outside of those working in special lines, and even these are undetermined. It is, therefore, very important, as Dr. Townsend suggests, that this matter be discussed at this time, in order that we may get our minds clear on this subject.

\section{THE TREATMENT OF UNCINARIASIS.*}

THOMAS A. CLAYTOR, M.D.

Professor of Materia Medica and Therapeutics, Columblan Un1versity ; Physiclan to Garfield and University Hospitals. WASHINGTON, $\mathrm{b} . \mathrm{C}$.

The treatment of uncinariasis may be considered under three headings: First, the prophylactic treatment, or that directed toward the prevention of infection by the hook-worm; second, the means used to cause the expulsion of the parasite; third, the treatment of the anemia and debility resulting from the infection.

Prophylaxis is of very great importance, and is a subject which requires careful consideration. It seems probable that infection takes place chiefly through the mouth, either in infected water or food, or through contaminated hands, which carry the parasite to the mouth. Looss, however, has apparently proven that the larvæ may penetrate the skin. A possible entrance through the air passages has also been suggested, but does not seem probable.

A most important fact, and one which greatly simplifies the treatment of uncinariasis, is that there is no increase in the number of parasites in the intestinal tract of the victim. Each hook-worm is taken into the system in the larval state, and then develops into a mature parasite capable of producing eggs which must, however, be passed from the host before they can be hatched. Thus it seems probable that the removal of a sufferer from an infected locality to one free from infection would ultimately result in recovery, if the vitality had not been already too much taxed.

\section{MODE OF INFECTION.}

The eggs are deposited in the fecal evacuations, and are, under favorable circumstances, hatched in twentyfour to forty-eight hours. In the larval state they become scattered in water or soil, and are now ready to infect the host who swallows them, or whose skin they penetrate.

Since the primary infecting agents are to be found in the evacuations, the important question seems to be how best to insure their destruction before they become scattered in the surface soil.

Sandwith says "cleanliness alone is required," but this is not enough. Of course, washing the hands before eating would prevent a certain number of infections, but if we remember the possibilities of infection through the drinking water and in the use of raw vegetables, to say nothing of the theory which Looss has proven, of infection through the skin, it can readily be seen that washing the entire body several times a day would not suffice.

The first step, the all-important step, is toward the destruction of the eggs and larvæ. It is well known that the embryos can not live without air. In view of this fact, Sandwith ${ }^{1}$ suggested that infected villages should be compelled to dig trench latrines outside the villages, to use the latrines alone, and to cover them daily with earth. Thus the larvæ, deprived of necessary air, would

- Read at the Fifty-fourth Annual Session of the Anerican Medical Association, in the Section on Practice of Medicine, and approred for publication by the Executive Committee: Drs. J. M. Anders, Fronk A. Jones and W. B. Thayer.

1. Observations on 400 Cases of Ankylostomiagis, 1894. soon die and cease to be dangerous, and as a result the disease would in time be stamped out.

Sandwith would also insure the death of the embryos by exposing the latrines to the sun's heat, as they have been known to live in shaded earth contaminated by feces for more than six months. Giles (quoted by Sandwith) recommends ploughing the infected surroundings of. villages, so as to bury the embryos.

Stiles $^{2}$ has adrocated practically the same thing as has Sandwith for the localities in our southern states where uncinariasis is known to exist. He suggests the use of suitable water-tight receptacles for feces, which should be emptied frequently; that the people should be taught the necessity of making use of these receptacles at all times, instead of defecating at any convenient spot, where the eggs hatch and the larvæ become disseminated through the surface earth.

This education of the people can only be accomplished by the family physician. No matter what care is taken or efforts made to enforce such regulations, the stamping out of this serious disease is going to be a difficult and tedious problem, simply because its occurrence is almost exclusively among the most ignorant of our people. The discharges might be burned or disinfected by chemical agents, but these methods are not practical in the country districts where uncinariasis prevails. To me the use of trench latrines would seem to be the most practical method of disposing of the infected feces, as it would necessitate very little trouble and no expense, items which would naturally appeal strongly to the people.

THE MEANS USED TO CAUSE THE EXPULSION OF THE PARASITE.

As an anthelmintic in uncinariasis thymol has almost entirely displaced all other remedies. (The credit of discovering the cure of ankylostomiasis by thymol seems to be due to Bozzolo, in 1880.-Sandwith.) The most popular method of its administration is as follows: The patient is given a purgative the day before, and is placed on liquid diet. Thymol is then given in 15 to 30 grain doses ( 1 to 2 grams) for four doses, at intervals of $t w o$ hours. If the bowels do not move spontaneously in twelve hours after the last dose, a purgative is given. If an alcoholic solution of thymol is used, the majority of the worms are passed in six to eight hours after the first dose. Therefore, the stools passed within this time are the most important to examine for the parasite. If alcohol is not used they are seldom seen earlier than twelve hours after the first dose.

Sandwith has found that 4 grams (3i) given in two doses of 2 grams (30 grains) each at 8 and 10 a. m. respectively, are quite as efficient as the use of 6 or 8 grams given in three or four doses at intervals of two grams given in three or four doses at intervals of col- $^{-}$ lapse. He also omits the primary purge as unnecessary and conducive to exhaustion, and gives a dose of sulphate of magnesia or castor oil two hours after the second half of the thymol. It has been found best not to repeat the treatment more frequently than once a week unless there should be some specially urgent reason for a rapid expulsion of the parasites. This method and dosage, I think, will be found most satisfactory.

The fact that thymol is poisonous must not be lost sight of, and the patient should be carefully watched. Some authors have cautioned against the use of alcohol, ether, turpentine, chloroform, oil, glycerin and certain

2. Report on the Prevalence and Geographical Destruction of Hookworm Disesse in the United States, 1903. 
alkaline solutions during the administration of thymol because of its solubility in these media, thereby increasing the danger of rapid absorption and consequent poisoning. Other writers, however, advise the use of alcoholic stimulants, on the ground that the depressing effect of thymol is best combated by this remedy. No less an authority than Sandwith says: "Warned by the death of at least one of my patients immediately after digesting the thymol, I have always administered to feeble men 25 grams of brandy with each 2 grams of thymol, with the happiest results."

Stiles and Pfender in 1902 performed a number of experiments on dogs with thymol in various forms. From these experiments Stiles concludes by saying: "I am afraid of the use of alcoholics per os during treatment, and in the case of weak patients I should be more inclined to use a stimulant hypodermically than run the risk of dissolving the thymol too rapidly, or in too great quantity at one time."

Since thymol is the accepted anthelmintic in the treatment of uncinariasis, and as there seemed to be some difference of opinion as to the safety of its use in alcoholic solution, I instituted a short study of its action on dogs. I regret to say that time did not allow of as complete an investigation as I would have wished before the presentation of our conclusions before this Association. I hope, however, that a few facts of value have been determined.

The work has been carried on by E. E. Butterfield, a fourth-year medical student in the Columbian University and pathologic interne at Garfield Hospital, to whom I wish to express my thanks.

\section{EXPERIMENTAL WORK WITH THYMOL.}

Thymol was administered in powder, in alcoholic solution, rendered bland by the solution of olive oil, and hypodermically. Space does not allow of a discussion of previous observations upon the physiologic action of thymol; it suffices to say that our results did not agree in all respects with those of other investigators.

Thymol given in dry powder in small doses (2 grams) caused salivation, and later slight diarrhea. No marked or constant effect upon the temperature, pulse or general condition. Large doses (5 to 20 grams) cause profuse salivation, later vomiting, anorexia, disinclination to move unless aroused, diarrhea and slight elevation of temperature $(0.5 \mathrm{~F}$. to $1.0 \mathrm{~F}$.), the latter appearing six hours or longer after the dose and lasting from twenty-four to forty-eight hours. Prolonged feeding in ascending doses of $2,3,4,5,6,7,8,9,11$, 14 and 20 grams during four weeks caused no loss of weight, the weight of the dog on which this experiment was tried having risen from 22 to 26 pounds during the course of treatment. Eleven grams of the dry thymol is, apparently, not a dangerous dose to dogs of 22 pounds or more, if given carefully and after a meal. Four dogs were used in these experiments without a death.

Thymol Given in Alcoholic Solution.-Alarming symptoms immediately following the administration of the alcoholic solution of thymol to dogs, seem to be almost always, if not invariably, due to aspiration of the irritating solution into the lungs. In seven dogs to which an alcoholic solution of from 2 to 10 grams was given an extensive consolidation of lung tissue was the only significant lesion.

If aspiration of the alcoholic solution of thymol occurs the symptoms are immediate. In a few seconds the dog staggers, seems unable to stand, and in a few minutes develops general convulsions with unconsciousness. The temperature falls 1 to $3 \mathrm{~F}$., pulse and respiration become rapid, pupils are widely dilated, and respond sluggishly to light. Convulsions last from five to fifteen minutes after consciousness is regained, the temperature rises slowly, and in from six to twenty-four hours reaches a point 1 to $2 \mathrm{~F}$. above the normal. This elevation persists for a number of days. A persistent cough develops, a fremitus may be felt over the lungs, and râles are heard. If recovery takes place the dog regains his appetite in one or two days, the cough gradually abates and the temperature falls to normal. This occurred in two out of seven cases. In some cases there is persistent cough, elevation of temperature, loss of appetite and emaciation which would probably end fatally if allowed to do so. Three out of seven presented these symptoms, but were killed on the third to the tenth day after dosage, extensive lung lesions being. found at the autopsy. Of the remaining two, one died almost immediately, the bulk of the dose having entered the lungs, none being detected in the stomach (the stomach tube had accidentally entered the trachea). The other, though not very sick, was killed four hours later to ascertain the early changes in the gastrointestinal tract.

Of the seven dogs experimented on, two recovered, and at the end of three weeks were apparently well, save for a slight cough; three would probably have died; one died; the remaining dog would undoubtedly have recovered, as the lung lesions were not extreme.

In conclusion it may be said that doses of 2 grams or more in alcoholic solution are unsafe for dogs, owing to a tendency to aspiration pneumonia.

Thymol in Alcoholic Solution Rendered Bland by the Addition of Olive Oil.-To avoid the irritating effect of the alcoholic solution, the drug was dissolved in alcohol, and enough oil added to render the mixture non-irritating, as tested by taste. Four grams were then given successfully to dogs of 16 to 17 pounds, without depression or fall of temperature. Six grams were given to a 17 pound dog, with a resulting fall of 2 degrees of temperature four hours after the dose was given, and lasting for six days, with depression, anorexia, diarrhea with bloody stools, emaciation (8 pounds lost) and coldness of the surface and extremities.

The autopsy, performed six days after the dosage, showed extensive subacute gastrointestinal inflammation with only two pea-sized areas of congestion at the base of the left lung.

Conclusions: Doses of 2 to 4 grams of thymol in alcoholic solution rendered bland with oil are safe for dogs of 17 pounds or more.

Thymol Hypodermically.-One 16-pound dog was given an alcoholic solution hypodermically to determine whether any toxic symptoms would develop from this manner of administration.

Ten c. gm., 0.20 gm., 1.00 gm. and 1.00 gm. repeated were given at intervals of one or two days without any apparent effect, except inflammatory swelling at the site of injection. Autopsy was performed one week after the last dosage. No pathologic lesions were found, except congestion of the lungs, which may have been produced during the process of anesthetization, which, in this case, was attended with considerable difficulty.

Autopsies on Eight Dogs.-Microscopic examination of the viscera of eight of the dogs to which thymol had been administered showed no changes which were not attributable to the local irritative effect of the drug. The liver, spleen, kidneys and heart muscle were normal. The changes in the lungs varied from intra-alveolar hemorr- 
hage to bronchopneumonia in cases where aspiration had occurred. The condition of the stomach and intestines varied from mild congestion to marked inflammation, most intense after the administration of the drug in alcohol and oil; least intense after the dry. The probable reason why the inflammation was not so marked after the administration of the simple alcoholic solution was that it was so irritating that much of it was drawn into the lungs by the violent coughing it caused. In no instance were albumin or casts found in the urine at autopsy.

\section{CONCLUSIONS.}

1. The alcoholic solution of thymol is dangerous to dogs, even in small doses, chiefly because of its tendency to produce inflammation of the lungs by aspiration.

2. The alcoholic solution, even when rendered bland by the addition of oil, is dangerous in doses of more than 4 grams to dogs of 16 or 17 pounds, because of its irritative effect upon the gastrointestinal mucous membrane.

3. In dry powder the drug may be given to dogs in large doses without serious results, no depression, no fall of temperature.

4. Gram doses given hypodermically cause only local inflammation.

5. There is probably no danger in giving 2 to 4 grams, followed by 1 to 2 tablespoonfuls of brandy, to an adult human being, if followed in a few hours by a purgative such as castor oil or magnesium sulphate.

6. It is probable that the alcoholic solution obtained in this way would be more efficacious against the parasites, as seems proven by their much earlier appearance in the stools after the use of the alcoholic solution.

\section{THE NUMBER OF DOSES REQUIRED TO EFFECT A CURE.}

As to the number of doses of thymol which are usually required to effect a cure, I can not do better than to again quote Sandwith, who says: "In 184 patients I was able to prove the absence of worms by giving at least two, and sometimes more, doses of thymol after the last appearance of the parasite in the stools. The average number of doses required proves to be 2.6. Forty-two men were cured after only one dose, fifty-eight after two, forty-two after three, twenty-five after four, nine after five, and four after six doses of thymol. Two cases required seven attacks, and two others eight."

In my own case, after giving thymol at least nine times in a period of nine weeks in two doses of 2 grams each, separated by two hours, and followed by a purgative, there were found at the autopsy twenty-nine worms in the small intestine. This serves as a warning that in some cases the parasites are extremely difficult to exterminate, and require a prolonged course of treatment. The stools should be examined weekly for eggs, and as long as they are present the thymol treatment should be continued.

As to the resulting anemia and debility, a general tonic treatment with iron and abundance of good food and fresh air are indicated.

1323 New Hampshire Avenue.

\section{DISCUSSION}

ON PAPERS OF DRS. STILES, SMITH AND CLAYTOR. *

DB. W. G. Harrison, Talladega, Ala.-After hearing Dr. Osler speak on this subject in his wards I felt sure that I saw some cases that were affected with hook-worm disease. When I came home a physician called attention to some suspicious cases at the Deaf, Dumb and Blind School. Several cases were found at the time the state medical association met with

* The paper of Dr. Stlles appeared in THE JoursaL July 18. The paper of Dr. Smith has not been furnished for publication. us, when Dr. Stiles was our guest. He confirmed the diagnosis in one of the four cases. A microscopic study of the case was not made until the early part of the last week. It was my purpose to treat the case with thymol, but at the suggestion of Dr. Stiles we gave them one or two remedies before resorting to thymol. These children were all in the schools. The youngest was 7 and the oldest only 18 years old. All were ex. tremely anemic. No blood counts were made. Hemoglobin in the lowest was 25 per cent., in the highest 43 per cent. We were careful to make an accurate temperature record. The temperature of each of these patients was not much elevated. In four it was over $100 \mathrm{~F}$., in three between 99.7 and $100 \mathrm{~F}$. In only one was $I$ able to demonstrate the malarial organism, so they could not have been due to latent malaria. In all cases there was a heart murmur, in three a systolic, in four an aortic. In four out of seven cases there was a marked pulsation of the vessels of the neck. It is rather an interesting point that Dr. Stiles himself recognized the disease in whites, but said he was not sufficiently familiar with its characteristics in the negro to recognize it. It was stated that it was likely one of the pupils would be affected with the hookworm, and many students. were examined and hook-worm disease found. Subsequently, in one of the schools, being unable to pick out the likely subjects, $I$ asked the superintendent if there were any who appeared to be especially lazy, or any pupils who would not play, who would not take exercise, or frolic, or run or jump. He answered: "Yes, I have two boys who are so lazy that I can not get them to do anything." I examined those two and found the eggs in both. Three out of the seven cases I examined were among the blind. I saw two cases among the mutes. The peculiarity about the eyes was noticed. I had heard Dr. Welsh speak of the "stare." As soon as we were inside I said: "Doctor, I guess you will find the stare." Subsequently we went through the carpet factory and found in three out of seven cases the stare.

The treatment of these seven cases was as follows: Two were given four grains of calomel every three hours for four doses, and after a couple of days Epsom salts were administered. Twenty-four hours later the worms could be found in the stools. Then, for each patient, for two days, thymol was given in 15-grain doses at intervals of three hours: On the following morning there were. found in the stools from 40 to 50 worns. The above was the treatment given in two cases. The third case was given 10 grains of salol every four hours for four days. No worms were found in 24 hours. In two days 10 grains of thymol were administered, and this patient passed 28 worms during the next 24 hours. In case four calomel, grains four, and santonin, grain one, were given every three hours until four doses were given. No worm inside of 24 hours. The next day two doses of 15 grains of thymol were given, and then there were passed between 60 and 70 worms. Case five fasted two days by accident, and this patient passed two worms before any medicine was given. That patient was given 10 grains of salol and 4 grains of calomel and 1 grain of santonin every three hours until four doses were given. This patient then passed 10 or 12 worms. On the succeeding day this patient was given 3 grains of thymol.every three hours until three doses were taken, and on the following morning there was found in the stool 27 worms. Case six was given 15 grains of thymol every three hours for two days, and this patient passed a large number of worms, over 250 , and probably four times as many more. Case seven had an incomplete record. He passed many worms.

Dr. E. D. Bondurant, Mobile, Ala.-My attention was first directed to the subject under discussion by a fellow practitioner in Mobile, Dr. W. T. Henderson, who told me of three cases which he had seen, and which from the peculiar edema shown he believed to be uncinariasis. The feces from those cases were submitted to me for microscopic examination, and in all of them I found the ova of uncinaria. Since then $I$ have succeeded in interesting a number of my students and other doctors in the study of the disease, and have had a large number of fecal specimens sent to me for diagnostic ex amination. In this way $I$ have already diagnosed more than 
fifty cases of uneinariasis in Alabama, chiefly from the counties of Mobile, Monroe, Escambia, Covington and Crenshaw, the greatest prevalence seeming to be in the sandy pine hill country west of the city of Mobile. In south Alabama there can be no question that the disease is more common on the sandy land than in the black lands or on dry formations. Uncinariasis is surely a very common complaint in our part of the world. It is rather a significant fact that in every instance in which uncinariasis has been suspected and fecal matter submitted for miscroscopic examination, I have been able to demonstrate the presence of hook-worm ova.

Being a neurologic specialist I have not had very great opportunity of studying the symptomatology and treatment of this disease, but during the past four months I have had to deal with uncinariasis as a complication or possibly causative factor in sundry eases of nervous disease. These patients, six or seven in alI, have shown the anemia, listlessness and general mental and bodily enfecblement already dwelt on by the authors of the papers under consideration, and all of them have been treated with thymol. The usual dose has been 60 grains, given in six capsules within two hours' time, preceded by calomel and followed by a saline. The results have been favorable without exception, numbers of worms being expelled and subsequent rapid improvement shown in the general condition of the patient.

Attention should, however, be called to the possible dangers of these large doses of thymol. One of my former pupils wrote me that he gave an 8-year-old boy 60 grains and came near killing him, the child passing into a conditon of coma with spasmodic movements and sceming at the point of death for hours; he was cured of uncinariasis, however.

Regarding the number of worms present, I know of one case in which a patient passed 953 , and another instance, reported to me by Dr. Parker of Crenshaw County, in which "over 1,700 worms were counted, and there were more beside."

I have made careful examination of the blood in four cases, and in each one found a great diminution in hemoglobin percentage, with comparatively slight reduction in the number of red blood cells.

Now that Dr. Stiles has overwhelmed us with evidence and shown us how anybody can diagnose uncinariasis, we should not forget that the disease was recognized by Dr. Allen J. Smith ten years ago, when no one knew much about it, and its frequency was unsuspected.

DR. C. A. Morr, Mobile, Ala.-Unless one has had a case of uncinariasis one can not appreciate the great work accom. plished by Smith and Stiles. We in Alabama can fully ap. preciate it, because they have been so plentiful there. For a long time we were unacquainted with the disease and were treating for this, that and the other thing, and especially for malarial anemia. In fact, the poor little devils have taken iron enough to build battleships, and without any effect on the anemia whatever. Only when our attention was drawn to the true nature of the trouble did we get any good results. The first case in Mobile was recognized by Dr. Hender$80 \mathrm{n}$, and he made a diagnosis from the clinical symptoms only. The pulsations in the carotids, the intense anemia, the Iowered amount of hemoglobin, the systolic murmur, etc., are all such striking symptoms. How the anemia is accounted for I am at a loss to say; I do not know whether it is due to the loss of blood, or whether it is produced by the toxemia, or possibly the secretions of the worms themselves. It is hard for me to believe from the amount of the anemia which is so intense as in chlorosis and the loss of hemoglobin, etc., that it is due to anything so far attempted in the way of an explanation. But I am sure that many cases of so-called "idiopathic anemia" will be found to be due to some parasitic disease.

DR. G. W. McCaskey, Fort Wayne, Ind.-My experience is limited to but three cases seen in northern Indiana, occurring over one year ago. Two cases were in Syrians, and therefore imported, while the other was an American.

I wish to emphasize the importance of a routine examination of the feces, not only in hospital cases, as advocated by Dr. Stiles, but in private practice as well. For several years
I have been in the habit of making this routine examination of feces in practically all cases. A number of writers think that it would not be necessary to do this; Ewald, Einhorn and others think it is not necessary. In 1895 I read a paper advocating this routine examination of the feces, and $I$ have been keeping up such examinations ever since. It is not especially unpleasant; less so than sputum examination. My principal object in getting up to-day is to plead for this routine examination of the feces in all cases of obscure chronic nutritional diseases, in which, among many other things, important parasitic ora may be found at any time.

Dr. Claude A. Smith, Atlanta, Ga.-This disease is not especially confined to the sand belt; it is also found in the mountain regions. Through observations that have been carried on in Atlanta and adjacent territory, and especially by examination of students in the Atlanta College of Physicians and Surgeons and the Southern Dental College, I have traced the disease from the Carolinas to Texas.

Almost every country practitioner in the South sees every year any number of cases of anemia, and many of them of the profound type, which are often classed as malarial cachexia, and they have been treating these cases with quinin, iron, arsenic, etc., with only slight temporary improvement, and I have found many such to be cases of uncinariasis, and the treatment with thymol has given positive results, which were sometimes remarkable.

Since we have recognized in the South that this disease is so very common, the point which concerns us most at the present time is by what means does it reach the intestines. During my early observations I was inclined to the common belief that it is taken into the body in the drinking water, but later observations led me to the conclusion that the larvæ gain entrance through the skin of the feet, as first pointed out by Bently, producing the ordinary "ground-itch," which is so common in the South in the summer after a period of rainy weather. In fact, I have found that it is possible to locate cases of uncinariasis that you would not otherwise suspect by simply getting the history of the patient in regard to attacks of ground-itch, and $I$ have found that every person who has had an attack of ground-iteh within a period of seven or eight years has the uncinaria in his intestines.

My first experience with this disease was in Decenber, 1901, when, on making a postmortem examination on a case which had died at the Grady Hospital in Atlanta, I found over 300 of the uncinaria in the small intestine, and on looking up the history of the patient I found that he was from Florida and had never been out of the South. As this case was evidently contracted in the South I naturally concluded that it must be endemic, and through the kindness of Dr. S. H. Green I obtained the stools of twenty convicts who work in the damp clay in one of the large brickyards near Atlanta, and of these twenty $I$ found two cases of uncinariasis. These cases were reported by me at the session of the American Medical Association at Saratoga last year in the Section on Pathology and Physiology. Since that time I have found the disease to be so common as not to be practicable to keep a record of all cases.

The reason the full extent of the disease was not recognized sooner was because it is a disease of the country and not of the city, and it abounds under unsanitary conditions.

In regard to the systematic examination of the stools of all patients, as mentioned by Dr. Stiles, since the first case appeared at the Grady Hospital I have made a systematic ex. amination of the stools of all patients, and during this time have found only three cases in the hospital. The rules of the hospital require that all applicants for admission must reside in the city, and this accounts for the small number. As I have mentioned before, you can locate the cases with a fair degree of accuracy by securing a history of the patient with reference to attacks of ground-itch within seven or eight years.

Dr. James B. Herrick, Chicago-I wish to call attention to the fact that although this is a disease of the tropics, we in the North should keep our eyes open for imported cases. This last year Dr. Capps of Chicago observed and reported 
a case that came from Panama, and who reached Chicago after a prolonged stay in the hospital in New Orleans. In this case it is interesting to note the occurrence of 13 per cent. eosinophilia with very marked anemia, the large number of eosinophiles calling attention to the possible existence of an intestinal animal parasite.

Dr. WM. Britr Burns, Memphis, Tenn.-I would like to refer to two cases which did not show uncinaria. The first case was an engineer who had been in a logging camp, sometimes having occasion to drive teams through lakes of mud. I had examined him a number of times for the malarial parasite, which I found, and thought he had a malarial cachexia. The man came to me in Memphis three months ago. He had fainted and $I$ restored him in my office. He was then placed in the hospital. He had a very marked anemia. He did not remain long enough for me to make a complete blood count. The blood was found to contain the malarial parasite. He had no temperature at that time. The stools did not show uncinaria or other intestinal parasite. This patient had marked symptoms of sleepiness; he slept every time he sat down. He was an engineer at that time and when it was time for him to pull the cord attached to the whistle, on one occasion he raised his hand to pull the cord and went to sleep, and passed the station. He had some diarrhea alternating with constipation, and his bowels would gurgle, as he expressed it. He had some colic. The stools were white. I wished to get him for further examination, but could not.

The second case was a soldier from the Philippines who came to me with diarrhea, and he said that he had had it for some time, possibly a year. He had been in the hospital for six months. Coming on to the pathologic service six weeks ago I made several examinations of his stools. There were no malarial parasites found in the blood. The parasites that I found in the stools were of this character: (Blackboard demonstration.)

I would like to know more regarding the character of the stools in these cases of uncinariasis and of other parasitic diseases of the bowels. I would also like to know the condition of the spleen and liver, the skin and sclera, and also something of the pathology of the mucous lining of the gut, whether there is ulceration or much involvement.

Dr. Charles Wardell Stries, Washington, D. C.-With regard to ground-itch, I may say that this is exceedingly common in people affected with uncinariasis; but so far as I understand at present it is also found in people who have no hook-worm disease. I am informed by physicians of Georgia and Florida that practically every inhabitant of these states, except persons living in cities, have had ground-itch at one time or another. Undoubtedly uncinariasis infection may take place through the skin, as has been demonstrated experimentally by Looss of Cairo, Egypt. As to infection through the drinking water, I think this is possible, but the chances are that such method of infection is not so common as might be supposed, for the reason that the larve of hook-worms sink in water. Infection through cotton, as was suggested in connection with one case, I consider practically excluded on account of the dryness of the cotton.

Length of life of parasite: I have two cases where the parasites have lived six years, and one case six years and seven months, after the patients entered an orphan asylum.

Disinfection: I consider chemical disinfection of premises impractical. Yards may, however, be disinfected with fire, either by spraying burning oil or by scattering straw around and setting it on fire.

Number of parasites present: Dr. Harrison's case, which passed over 1,700 worms, is interesting, and so far as I know now holds the world's record. Ashford sent me about 1,400 worms from one case in Porto Rico.

Classification of the parasite: Dr. Smith has spoken of the validity of the American species. My studies on the anatomy of hook-worms have been interrupted, but it is now easy to foresee that the old genus Uncinaria will have to be subdivided. Worms like Anchylostoma duodenale and A. caninum form a distinct group characterized by the presence of re- curred hooks in the mouth; these parasites should be classi. fied in the genus Anchylostoma. The worms without buccal hooks, but provided with buceal lips, belong at present in the genus Uncinaria, but these forms may be divided into several subgenera, at least, which may eventually be given the rank of genera. The typical subgenus is Uncinaria, with $U$. vulpis as a type. Species with non-symmetrical bursa and with prominent buccal tooth, like Uncinaria radiata of cattle and $U$. trigonocephala of sheep, may be placed in Railliet's (1902) Bunostomum. Monodontus (Molin), 1861, should be based on $M$. semicircularis, and apparently presents, according to Molin, a symmetrical bursa with dorso-median ray of the usual type. Uncinaria anericana seems to take a very isolated position, with its buccal capsule more or less similar to that of Bunos. tomum, but with a symmetrical bursa in which the dorsomedian ray is of a very aberrant type. I believe we are justified in recognizing for this species a distinct subgenus (for which I would suggest the name Necator-a murderer), which may eventually be raised to generic rank. Uncinara lucasi is still different in certain characters, notably of the anterior end, and will probably call for the erection of a new subgenus. Agriostomum and Characostomum (Railliet), 1902, will probably stand as distinct genera.

The anemia: I agree with the view that it is difficult to explain the severe anemia and the nervous symptoms by the mere extraction of blood. In all probability the cervical glands of the parasites produce a poison which could be readily absorbed by the wounded mucosa of the intestine.

Diagnosis: As to the unpleasantness of a fecal examination, I frankly admit that I would much rather examine the feces than the sputum of a tuberculous patient.

Topography: I do not recall ever having stated that hookworm disease is confined to sandy soils. What I claim is, that it is pre-eminently a disease of sandy regions.

Color of stools: The color of hook-worm stools is frequently a peculiar reddish brown, a quite different shade from anything $I$ recall in connection with feces $I$ have examined in Washington. Still, this color is not constant even in very severe infections. Nor is the blotting-paper test constant; in fact, it should never be relied on to the exclusion of the microscopic examination, for the chance of error is too great. I suggest the test simply as a convenient confirmatory test in severe cases for physicians in the infected district who are unable to make the microscopic examination. Blood in the stools is by no means constant. It does, however, occur.

Dr. Allen J. Smith, Galveston, Texas-It should be kept in mind, in comparing the group of eases which $I$ have presented with the types of cases which have been mentioned in this discussion, that these instances represented a mild form, presented but few symptoms and were found in the course of a routine examination of a large group of persons undertaken because in some one or more of these persons it was certain the parasite existed. In the first and second of these cases the presence of an eosinophilia, found when searching for the malarial hematozoon, suggested the investigation of the stools for the uncinaria known to be present somewhere in the general group of students; and naturally the success of the search in these two early cases led to the thought of the value of the symptom as at least of suggestive force. In those cases of which I have records of the differential leucocyte count the proportion of eosinophiles ranges from 6 to 23 per cent.

There can be little if any doubt of the fact that one mode of acquirement involves the penetration by the embryonic worms in dirty water or in the mud adhering to dirty objects through the skin and tissues to their ultimate place of abode in the alimentary canal; while it is quite probable, too, that in many cases the entrance to the intestine is direct from drinking unclean water or eating dirty vegetables moist with damp earth. I do not feel that Dr. Stiles' view of the improbability of their transference by cistern water has included conditions which commonly prevail in the frequent over-ground cisterns found in many southern localities. There, is easy chance for entrance of the ova to such cisterns by their being blown with sand and dust to the house-tops, whence they may be washed 
by succeeding rains into the cistern. Sinking to the bottom of such receptacle they usually find what seems to me to be a not unsuitable surrounding for their incubation, providing they have retained their vitality, in the layer of sand or mud, mixed as it often is with charcoal commonly introduced into cisterns with a view of purification of the water; and as the faucets of these cisterns are placed close to the bottom it seems possible that the embryonic worms may be drained with the water used for drinking purposes or otherwise.

I also wish to lay some stress on the relationship which I believe may exist between this parasite and malaria. In six of the eight cases which I have mentioned there is a history of malarial complication, temporarily influenced by quinin, but invariably recurring after longer or shorter intervals. In several of these cases the demonstration of the malarial organism was unquestionable, but invariably was made only after repeated search and with more or less difficulty. While these malarial outbreaks were temporarily restrained by the administration of quinin, during the period of prevalence of the hook-worm in the intestine the malarial recurrences persisted indefinitely, the use of the quinin producing no permanent efiect. All this ceased after the parasites were removed from the intestine, subsequent administration of quinin being promptly and permanently followed by the disappearance of the malarial symptoms. It might be suggested that in an indirect way the intestinal parasites have an influence in bringing about a persistence of the malarial infectionprobably by lowering the natural powers of the system of the host in reaction and antagonism to some small residuum of the malarial infection after partial destruction by quinin, so that subsequently that residuum more surely may increase to a degree capable of producing the recurrence noted in such cases. With the removal of the worms there would seem to return the natural resistive influence of the body, and this joined with the potency of the quinin leads to complete and permanent destruction of the malarial infection. Doubtless there are other explanations possible for what in my experience is a very real relation of the character mentioned; but the idea suggested does not seem improbable. In such cases of an apparently malarial infection, with few organisms ordinarily recognizable, and these often missed, and with an irregularly recurrent type persisting after the use of quinin (although the latter may have been temporarily beneficial), I would urge that investigation be made for a possible complication by uncinariasis. If the latter be recognized I am confident that the experience $I$ have mentioned may be repeated, and that by first removing the intestinal parasites it will be found that a difficult and persistent malaria will become readily amenable to the ordinary administration of quinin. It adds but little difficulty to the labor of diagnosis. The hook-worm egg is one of the most easily detected of microscopic objects in the fecal material; there is no complicated technic required in the examination of the material, and probably five minutes' attention as an average will suffice for the added work.

The relation between hook-worm diseases in man and in the $\operatorname{dog}$ is an item in whieh I am satisfied that I have publicly expressed mistaken ideas. In the discussion of this matter in 1901 before the Texas State Medical Association, I commented on the similarity of our human uncinaria and the dog hook-worm, and I expressed some question as to a possibility of identity as well as a possibility of the dog being an agent in scattering the parasites where man might acquire them. From the work of others as well as from the clear differences I have since learned to recognize between these parasites I fully realize the mistake into which my ignorance then led me.

I am satisfied, too, of another mistaken suggestion I made at the same time, namely, of the possibility of incubation of the eggs in the intestine of an original host, and thus a chance of permitting a slight infection to increase to a serious degree and to make itself permanent. While $I$ am unable as yet to entirely set this idea aside as an occasional possibility, there is no doubt in my mind that it is essentially always untme. That it is not impossible I would infer from the fact that occasionally the ova in very fresh feces are found to contain well-formed embryonic worms; and that rarely even in the ova within a gravid female worm an embryo may be found. Of course, too, the segmentation seen in the ova ordinarily is evidence of some procedure of incubation; and should the egg happen to be retained unduly in some groove or in a mass of mucus adherent to the mucous membrane of the intestine, it might on rare occasions develop into the freed embryonic worm. It is impossible for me to indicate why this does not ordinarily take place. I haye noted in the incubation of ova in the fecal material that the process has proceeded more quickly and certainly at the ordinary room temperature than in the incubator at body temperature; and although there are probably other factors than mere tem. perature at work in this connection, it may be one element in restraining incubation in the intestine.

Dr. Thomas A. Claytor, Washington, D. C.-Regarding the elevation of temperature in these cases, it has been claimed that fever is unusual. I think, however, that this depends on the degree of anemia. In intense anemia, no matter from what cause, moderate fever of an irregular type is frequently seen.

Several of the gentlemen have spoken of symptoms by which they could diagnose uncinariasis-such as paleness of the skin, hemic murmurs, pulsation of the vessels of the neck, etc., which mean nothing but anemia, and hence are only suggestive. Without examination of the stools I do not believe a positive diagnosis can be made. It is an easy matter today to have such examinations made.

Regarding the dose of thymol to be administered, it is from 30 to 60 grains ( 2 to 4 grams). Sandwith, who has had a very large experience in the use of this drug, gives 30 grains at 8 o'clock, 30 at 10 and after two hours a purgative. In weak individuals he gives an ounce of brandy for every 30 grains of thymol.

It has been said that the American hook-worm is more readily gotten rid of than the old-world variety, because of the absence of hooklets in the former. In a case which $I$ have reported two months' treatment with thymol failed to entirely free the sufferer from the parasites, though they were of the American variety.

The weekly treatment with thymol should be continued until the eggs disappear from the evacuations.

The Danger of Corrosive Sublimate Solution as a Vaginal Douche.-It is strange that at the present time many medical men are found who continue to use corrosive solution for purposes of postpartum douching in spite of the clear demonstrations that have been made of its unsuitability and of its dangers, and in spite of the introduction of many safe and effcient substitutes. The use of this dangerous poison for postpartum douching is not alone confined to general practitioners, but it is used and recommended by many competent specialists. A case is recorded in an American contemporary in which a patient, aged 33, who had miscarried at the fourth month, was given a single vaginal douche consisting of a quart of 1 in 1,000 corrosive sublimate solution. The next day she exhibited all the characteristic symptoms of sublimate poisoning-gingivitis, salivation, and diarrhea, the symptoms became rapidly worse, and she died a fortnight later. Of course, an antiseptic can not be condemned because it is carelessly used with fatal consequences in a single case, and, if corrosive sublimate had anything to very strongly recommend it, it would be foolish to do so, but what are its recommendations? It destroys metal instruments; it is a most dangerous lotion to leave about a house; it is decomposed and rendered useless in the presence of much albumin; it roughens the hands of the operator, and constringes the mucous membrane of the vagina and vulva, and so tends to encourage the occurrence of lacera. tions of these parts; and, as the case to which we have called attention shows, and as many other reported cases show, its use is by no means free from danger. The obstetrician who uses it himself is courageous, but the obstetrician who recommends it for general use to others-nurses or students-is foolhardy.-Mledical Press. 